\title{
Characteristics of Acoustic Emission Signals Generated by the Contacts of the Selector
}

\author{
A. Cichon ${ }^{*}$, S. Borucki, D. Wotzka and M. Szmajda \\ Faculty of Electrical Engineering, Automatic Control and Computer Science \\ Opole University of Technology, S. Mikołajczyka 5, 45-271 Opole, Poland
}

\begin{abstract}
An important part of electric power transformer is the on load tap changer which enables for voltage regulation in the network. Due to high level of failure frequency of these devices it is necessary to look for new diagnostic methods which enable for theirs technical state estimation under transformer normal operating condition. Presently at the Electric Power Institute at Opole University of Technology there are works in progress aspiring to determine the application possibility of the acoustic emission method for on load tap changer diagnosis. Results of scientifically-research works aiming to characterize the acoustic emission signals generated by a separated selector are presented in the paper. Measurements have been performed in a setup with a model of on load tap changer type VEL-110-27 from ELIN. Acoustic emission signals generated by the selector have been analyzed in detail. Analysis of the time-frequency components of signals generated by on load tap changer has been carried out with the short time Fourier transform and continuous wavelet transform. Based on the comparison outcome of acoustic emission signals generated by the power changer and by the selector two measuring paths endowed with wide band contact transducers have been proposed for the practical application.
\end{abstract}

PACS: 52.80.-s, 43.28.Js

\section{Introduction}

A significant majority of on load tap changers (OLTCs) working in the country power system are constructions, in which the selector and the power switch compose two separate components. A switch of such construction was used in studies, whose results are presented in this paper. During operation of the selector there is a smooth movement of the contacts, by which acoustic emission (AE) signals of very small maximum value (as compared to the power switch) are generated. These signals also do not have such characteristic and repetitive structures. A very important selector parameter is the technical condition of his contacts, which directly affects one of the most important parameters of the transformer, namely the winding resistance. The practical exploitation often leads to damage of the movable contacts. Under the influence of these defects the clamping force between the movable and fixed contacts is reduced, which may lead to the occurrence of electrical discharges during the tap change or during normal transformer operation. The phenomenon of discharges formation, dangerous to the contacts, was used as a diagnostic indicator by the technical condition estimation of the selector by AE method [1-9]. Implementation of this evaluation is therefore possible during switching operations of the OLTC under load. The measuring result is shown in form of AE signal time runs

\footnotetext{
* corresponding author; e-mail: a.cichon@po.opole.pl
}

and its envelope, determined by means of the Hilbert transform absolute values, time-frequency spectrograms of power density spectra and continuous wavelet transform (CWT) scalograms.

\section{The measurement setup}

Measurements of AE signals generated during operation were performed on a test bench containing the complete OLTC system equipped with a power switch and a selector. Because of its large size, the study was conducted in transformer renovation workshop belonging to one of the distribution companies operating in the country. The main part of the system is a cylindrical measuring tank of $2.7 \mathrm{~m}$ and a diameter of $80 \mathrm{~cm}$. Its view is shown in Fig. 1a. Inside of the tank the complete OLTC system, consisting of a selector and a power switch, is mounted in the insulating sleeve. Installation of the power switch inside the insulating sleeve is carried out through a specialized winch. The view of the power switch during assembly is illustrated in Fig. 1c.

During the test, the entire measuring tank was filled with insulating oil. Switching was performed manually by forcing a coupling rotary motion. The measurements were performed at a rated current flow. In order to force the current a current source of voltage equal to $50 \mathrm{~V}$ was used, allowing for adjustable current to flow from 0 to $250 \mathrm{~A}$.

The measuring system is equipped with a broadband transducer WD type AH 17, which was attached to 


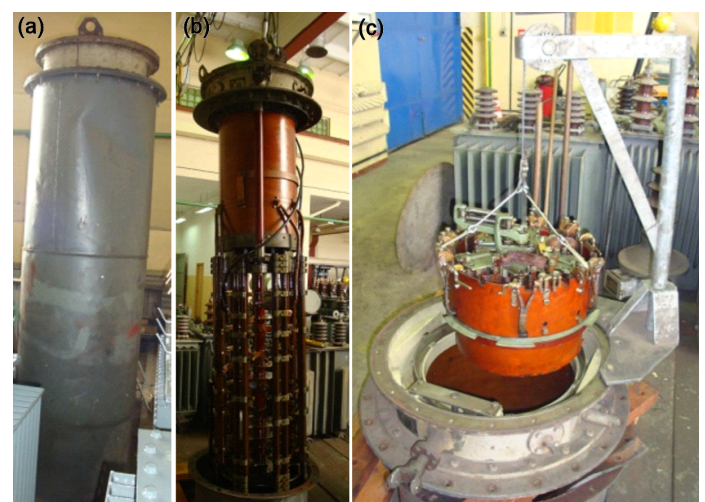

Fig. 1. View of the measuring setup: (a) measuring tank, (b) insulating sleeve and the selector, (c) assembly of the power switch in the insulating sleeve.

the outer wall of the tank using a permanent magnet. The transducer used is characterized by high sensitivity $(55 \mathrm{~dB} \pm 1.5 \mathrm{~dB}$ with respect to $\mathrm{V} /(\mathrm{m} \mathrm{s})$ and wide bandwidth from $100 \mathrm{kHz}$ to $1 \mathrm{MHz} \pm 10 \mathrm{~dB}$. This transducer is equipped with a differential measuring of AE signals. This allows for elimination of interfering signals, which appear under the influence of the electromagnetic field acting on the transducer and the measuring cable [1-3]. This is particularly important during measurement of the current flow during the OLTC switching process. The received AE signal was amplified in the preamplifier system of type $2 / 4 / 6$, which enables step gain adjustment: 20 , 40 and $60 \mathrm{~dB}$. During the measurements the preamplifier was set for $20 \mathrm{~dB}$, in some cases the gain was increased to $40 \mathrm{~dB}$. From the preamplifier the AE measuring signal was transmitted to amplifying circuit adjusted to the gain value of $20 \mathrm{~dB}$. In the system a band-pass filter with cut-off frequencies 10 and $500 \mathrm{kHz}$ was applied.

\section{Analysis of measurement results}

At the beginning, the so-called "fingerprint" for the selector operating without defects was determined. For this purpose rated current flow was forced between a pair of selector contacts. During measurement the AE signals were registered with a broadband transducer mounted in the middle of the measuring tank. View of the movable and fixed selector contacts used in the study is shown in Fig. 2.

Time run and the Hilbert transform absolute value of the AE signals recorded for the full switching cycle of the OLTC in the system with no selector defects is shown in Fig. 3.

AE signals characterizing the selector operation are in the first part of the course, in the range $0-1.5 \mathrm{~s}$. In the considered case they are characterized by low value of the maximum, which does not exceed $0.1 \mathrm{~V}$ and with a low prevalence, which has been in detail presented in Fig. 3b. Temporary structures that appear in the signal

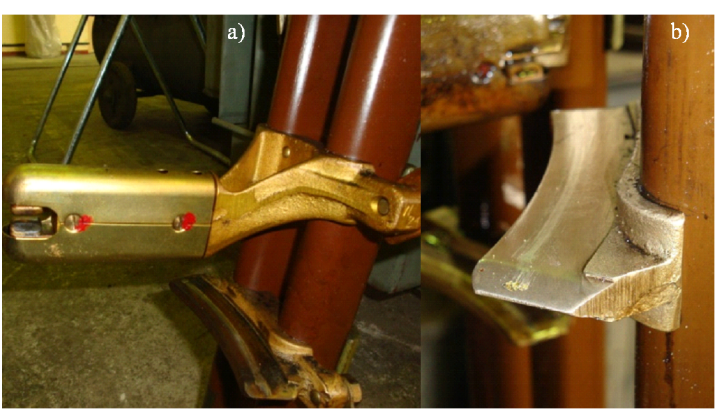

Fig. 2. General view of the selector contacts: (a) movable, (b) fixed.

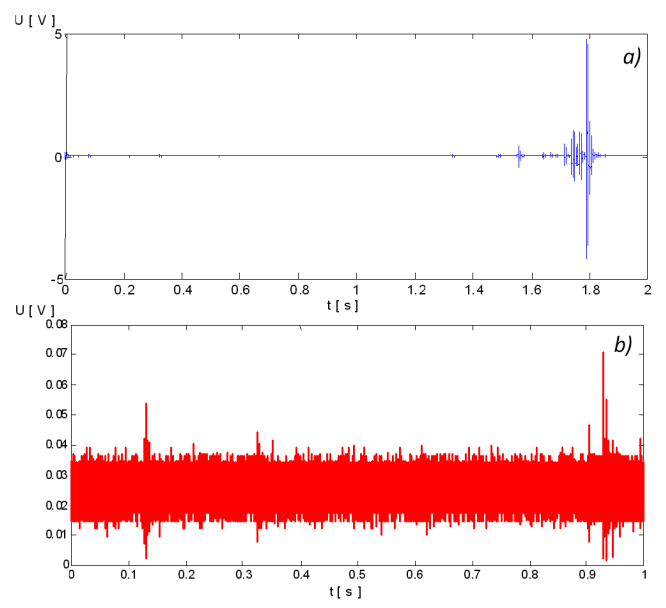

Fig. 3. Time run (a) and Hilbert transform absolute value (b) of AE signals generated by selector operating without defects.

after $1.5 \mathrm{~s}$, are generated by the OLTC mechanical system and operation of power switch.

In Fig. 4 power density spectrograms of the AE signals registered during full switching cycle of the OLTC are presented.

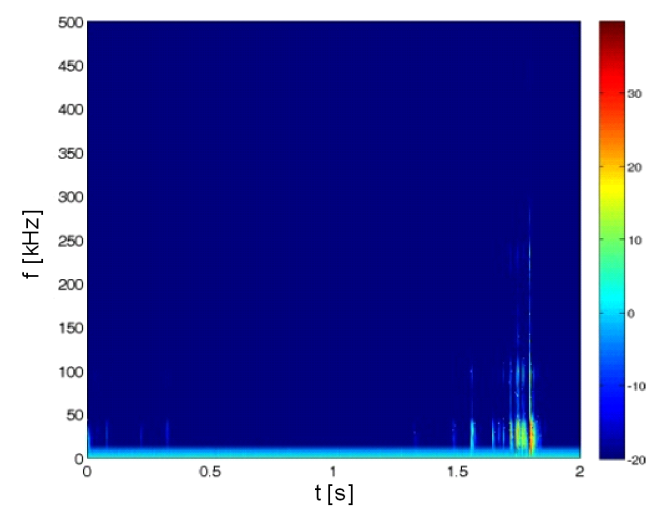

Fig. 4. Spectrogram of the power density spectra of AE signals generated by the selector operating without defects. 
In the time interval characterized by the selector operation one can see single structures, for which the dominant frequency band does not exceed $50 \mathrm{kHz}$. They are characterized by low value of power density and low intensity. Analyzing the frequency components, corresponding to the power switch operation that appear in the range 1.5-2.0 s, one can conclude that in the band of higher frequency attenuation of components above $250 \mathrm{kHz}$ occurred. This suppression may be due to a considerable distance between the transmitter and the power switch. For a detailed analysis of the structures of time-frequency AE signals generated by the selector, CWT scalograms for a time period limited to $300 \mathrm{~ms}$ was determined. Results of this analysis are shown in Fig. 5.

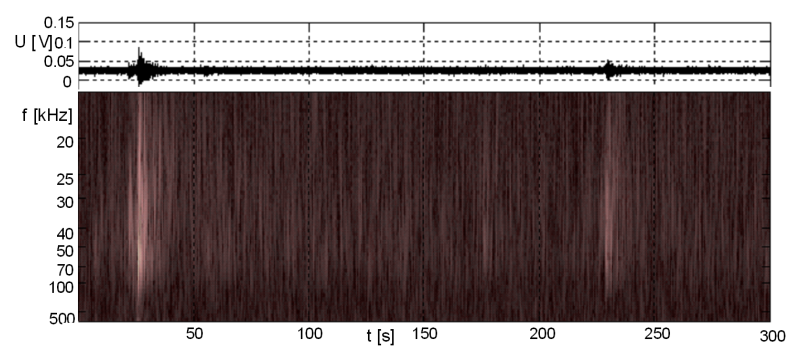

Fig. 5. A CWT scalogram of AE signal generated by operation of the OLTC under load with a selector without defects.

In the selected portion of the AE signal, characterizing the selector operation, temporary structures indicate a low maximum value, which does not exceed $0.1 \mathrm{~V}$. The intensity of acoustic events is small. The events correspond to coherent structures, whose dominant frequencies band ranges from 0 to about $150 \mathrm{kHz}$. Generation of AE signals with such a small value of the maximum may be due to the phenomenon of mutual contacts friction and sparse discharges of low energy that can arise during mutual selector contacts friction.

Due to the destructive effect of the electric arc, damage to the movable contact can lead to more serious consequences. The contact force between the movable and fixed contacts, depending on the design of the selector, is generated by various types of spring presser. Their mechanical damage reduces the clamping force between the contacts and also increases the contact resistance during both the switching process and normal operation. While contacts move under flowing load current it comes to a high-arcing and burning of contacts. This phenomenon was modeled by reducing the clamping force of springs in the selector contacts system. One pair of contacts was studied sequentially by forcing the rated current flow of $250 \mathrm{~A}$. After the measurements visual inspection of the contacts was performed during which, significant mechanical damages of both movable and fixed contacts were found. View of the contacts after the measurements is shown in Fig. 6.

In Fig. 6 there are visible intensive signs of fixed contact burns, which lead to its deformation and total loss

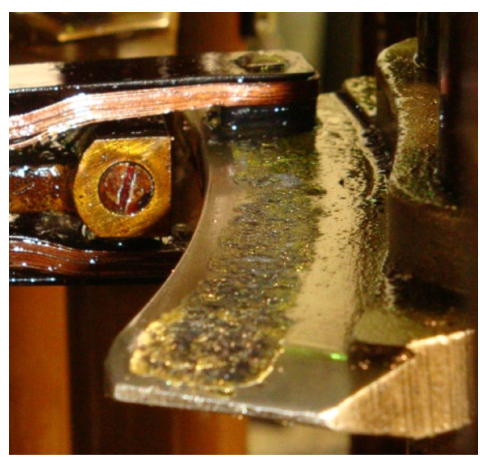

Fig. 6. View of selector contacts after measurements with damage on movable contact.

of switching capacity. The results of AE signal measurements in time domain is shown in Figs. 7 and 8 . Visible in the course shown in Fig. 7 acoustic events are characterized by a large maximum value and intensity. They occur practically in the whole signal registration time.

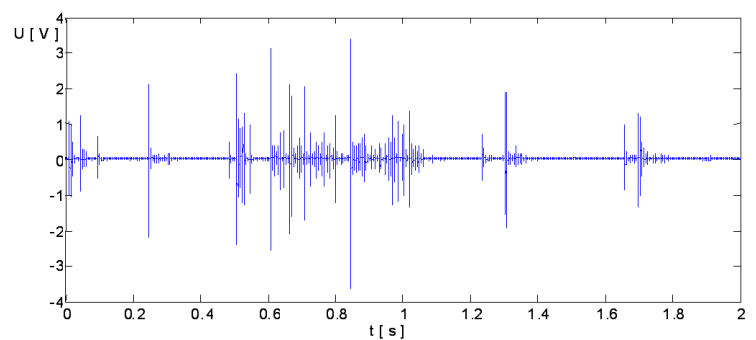

Fig. 7. Time run of AE signals generated in a setup with damaged movable selector contact.

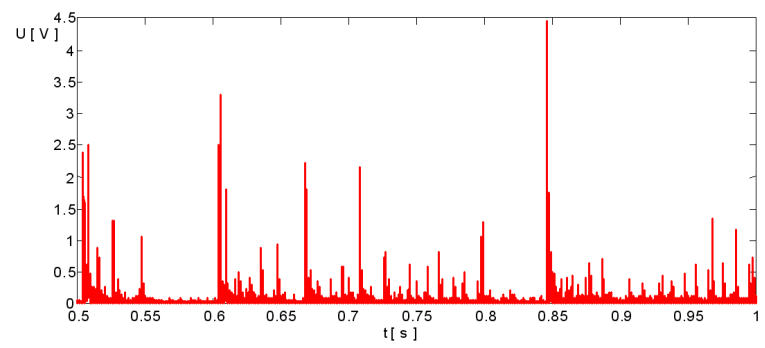

Fig. 8. Course of the Hilbert transform absolute value of AE signals generated in a setup with damaged movable selector contact.

The relatively large maximum value of the recorded signals indicates a high discharge intensity occurring almost throughout the whole selector operation cycle. Increased frequency of acoustic events is in the time interval $0.5-1 \mathrm{~s}$. The structures occurring in this period are characterized by varying maximum values taking place in the range from 0.5 to $4.5 \mathrm{~V}$. The spectrogram of power spectral densities of AE signals with the full selector operation cycle is shown in Fig. 9. 


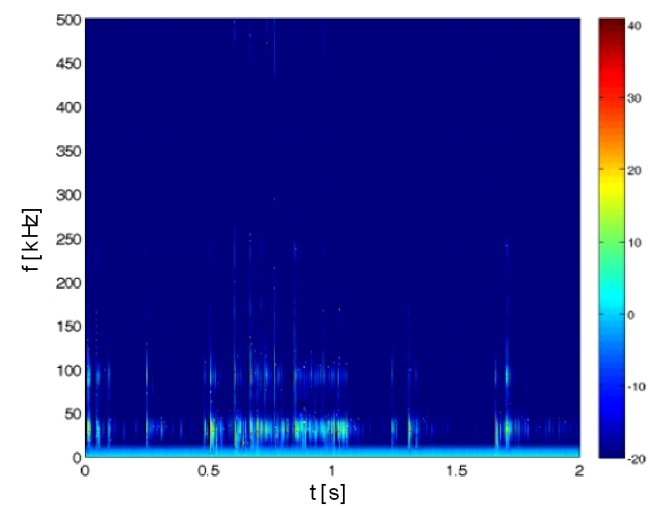

Fig. 9. The spectrogram of power spectral densities of AE signals generated in a setup with damaged selector movable contact.

The presented time-frequency image is characterized by a high number of structures containing frequencies in the range $20-150 \mathrm{kHz}$. In the spectrogram presented (Fig. 9) there are also structures visible, for which the dominant frequency band is wider and includes components from 20 to about $250 \mathrm{kHz}$. The wider frequency band occurs especially for component characterized by a higher signal power density value in the time domain. A detailed time-frequency picture for the interval shortened to $300 \mathrm{~ms}$ is shown in form of CWT scalograms illustrated in Fig. 10.

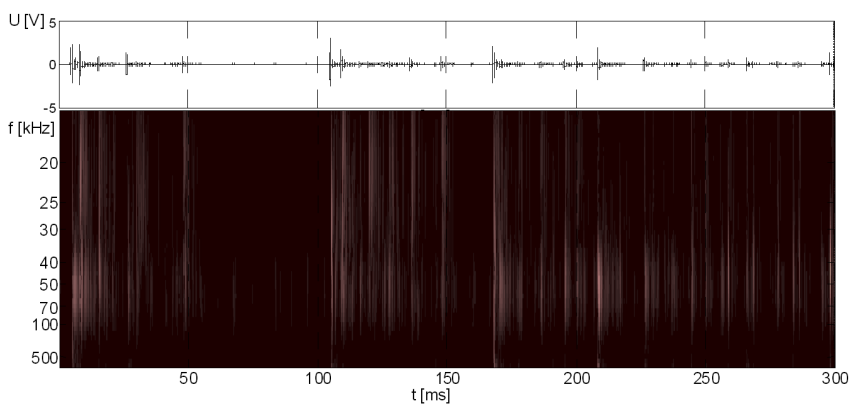

Fig. 10. A CWT scalogram of AE signals generated in a setup with damaged movable selector.

The analyzed AE signal is characterized by a large number of acoustic events. In the observed wavelet structures, corresponding to these events, one can identify a dominant frequency band, which is characterized by long period of occurrence. This band includes components from the range $0-150 \mathrm{kHz}$. Structures containing frequencies above $150 \mathrm{kHz}$ are characterized by smaller values and shorter duration. The range of occurring dominant frequencies indicate that in the analyzed AE signal the significant part of the acoustic events was generated by electrical discharges formed between the contacts.

\section{Summing-up}

As a result of the works presented in this paper it has been shown that using of modern signal processing methods in the time and time-frequency domains, it is possible to apply the AE method for the OLTC technical condition estimation of construction with a separate selector and power switch, during its normal operation.

\section{Acknowledgments}

The research work is co-financed by the National Centre for Research and Development.

\section{References}

[1] A. Cichon, Przeglad elektrotechniczny 88, 22 (2012) (in Polish).

[2] A. Cichon, Acta Phys. Pol. A 116, 290 (2009).

[3] A. Cichon, P. Frącz, D. Zmarzły, Acta Phys. Pol. A 120, 585 (2011).

[4] J.J. Erbrink, E. Gulski, P.P. Seitz, R. Leich, Electr. Insulat. 111, 252 (2008).

[5] C. Tyszkiewicz, T. Pustelny, Opt. Appl. 34, 507 (2004).

[6] T. Pustelny, I. Zielonka, C. Tyszkiewicz, P. Karasinski, B. Pustelny, Opto-Electron. Rev. 14, 161 (2006).

[7] M. Foata, R. Beauchemin, C. Rajotte, IEEE ESMO 17, 293 (2000).

[8] E. Rivas, J.C. Burgos, J.C. García-Prada, IEEE Trans. Power Delivery 24, 687 (2009).

[9] E.F. Simas-Filho, IEEE Trans. Power Delivery 25, 931 (2010). 